\title{
Biological Agent Genus and Species
}

National Cancer Institute

\section{Source}

National Cancer Institute. Biological Agent Genus and Species. NCI Thesaurus. Code C158309.

The genus and species of the biological challenge agent used in the study. 